\title{
Political Ideology of Donors and Attribution Messages in Charity Advertising: An Abstract
}

\author{
Younghwa Lee and Sukki Yoon
}

\begin{abstract}
Two experimental studies demonstrate that advertising messages emphasizing individualistic or structuralistic attribution can have varying effects on people with different political ideologies. We found that liberals tend to more positively evaluate the ad and more likely participate in a charity event when the ad message includes structuralistic attribution (e.g., the society caused homelessness), while conservatives tend to more positively evaluate the ad and more likely participate in a charity event when the ad message includes individualistic attribution (e.g., the homeless people caused their homelessness). We further shows that the ad evaluation-attitude toward advertising-plays a mediating role between the ideologyattribution fit and participation likelihood in the charity event.
\end{abstract}

References Available Upon Request

Y. Lee $\bullet$ S. Yoon $(\bowtie)$

Bryant University, Smithfield, NC, USA

e-mail: ylee6@bryant.edu; syoon@bryant.edu 